\title{
Cryptosporidium in asymptomatic children in Southern Xinjiang, China and the potential of zoonotic transmission
}

Tian Wang

Tarim University

Zilin Wei

Tarim University

Ying Zhang

Tarim University

Qiyuan Zhang

Tarim University

Longxian Zhang

Henan Agricultural University

Fuchang Yu

Henan Agricultural University

Meng Qi ( $\sim$ qimengdz@163.com )

Tarim University

Wei Zhao

Wenzhou Medical University

\section{Research}

Keywords: IbA9G3, IdA14, IfA12G1, DNASTAR, GENEWIZ

Posted Date: May 25th, 2021

DOl: https://doi.org/10.21203/rs.3.rs-551246/v1

License: (a) This work is licensed under a Creative Commons Attribution 4.0 International License.

Read Full License 


\section{Abstract}

Background: Cryptosporidium is a primary cause of diarrhea in children globally. However, there is limited information on the prevalence and genetic characterization of Cryptosporidium in children in Xinjiang, China. This study aimed to assess the genetic characteristics and epidemiological status of Cryptosporidium in asymptomatic children in Southern Xinjiang, China.

Methods: A total of 609 fecal samples were collected from kindergartners aged 2-6 y from 11 counties of Southern Xinjiang, China. We used nested PCR amplification of partial SSU rDNA gene to screen the samples for Cryptosporidium spp. The isolates containing $C$. parvum and $C$. hominis were further subtyped by a $60-\mathrm{kD}$ a glycoprotein ( $g p 60$ ). We used MEGA7 to construct a phylogenetic tree to study the genetic relationship between the gp60 subtypes of these two species via the Maximum Likelihood method based on the Tamura-Nei model.

Results: Only $1.3 \%$ (8/609) of asymptomatic children were confirmed as infected with Cryptosporidium with $2.0 \%(6 / 299)$ infection rate in boys and $0.6 \%(2 / 310)$ infection rate in girls. Three Cryptosporidium species were identified including $C$. felis $(37.5 \% ; 3 / 8)$, C. hominis $(37.5 \% ; 3 / 8)$ and $C$. parvum $(25.0 \%$; 2/8). Three $C$. hominis subtypes (IbA9G3, IdA14 and IfA12G1) and two $C$. parvum subtypes (IIdA14G1 and IIdA15G1) were also found.

Conclusions: This study was the first to identify the presence of cryptosporidium in asymptomatic children in Southern Xinjiang, China. The presence of zoonotic C. parvum subtypes IldA14G1 and IIdA15G1 indicates possible crossspecies transmission of Cryptosporidium between children and animals.

\section{Background}

Cryptosporidium spp. are obligate intracellular protozoan parasites, which can be found in all classes of vertebrates [1]. Cryptosporidium infects the gastric and intestinal epithelial cells leading to self-limiting diarrhea or asymptomatic symptoms in healthy people. However, infection can be fatal amongst immunocompromised individuals [2]. Cryptosporidium infection leads to stunted growth and weight loss, among other features. Cryptosporidiosis accounts for approximately $30.0 \%-50.0 \%$ global deaths per-year in children $<5 \mathrm{y}$ of age and is the second leading cause of diarrhea-related fatalities in children after rotavirus [3]. Cryptosporidium can be transmitted via various routes, including direct contact with infected individuals/animals, or uptake of contaminated food/water [4].

Cryptosporidiosis is a complex genus in which a total of more than 40 species and over 40 genotypes (yet to be assigned a species) have been formally described, all with high genetic diversity $[5,6]$.

Molecular studies have demonstrated that human cases of cryptosporidiosis are caused by minimum 20 different species. The dominant subtypes include $C$. hominis and $C$. parvum followed by Cryptosporidium meleagridis, $C$. ubiquitum and $C$. viatorum. Meanwhile, $C$. canis, $C$. felis, $C$. cuniculus, and $C$. andersoni, in addition to three Cryptosporidium genotypes (horse, chipmunk I and skunk) cause disease in humans 
and are zoonotic [7]. Various gp60-targeted subtyping tools have been designed to assess the importance of zoonotic transmission, using comparative analyses of human and animal samples $[7,8]$.

In China, recent studies have focused on Cryptosporidium in children in Central and Southern China but molecular detection methods have been used in no more than three reported studies [9]. Four species of Cryptosporidium, including $C$. meleagridis, $C$. hominis, $C$. canis, and $C$. felis have been identified in children from China. Amongst them, $C$. hominis is the predominant species and four subtype families including $\mathrm{la}, \mathrm{lb}, \mathrm{Id}$ and $\mathrm{Ig}$, are present within the $C$. hominis isolates [9]. Nevertheless, the species and subtypes of Cryptosporidium in children in China remain poorly defined, particularly in specific provinces or regions such as in Xinjiang Uygur Autonomous Region (XUAR), where a number of zoonotic species of Cryptosporidium are distributed in local animals. We therefore propose that the scale of infection by Cryptosporidium in children has been underestimated in China. This population poses a significant threat if the infections continue to be ignored.

In this study, we investigated the epidemiology of Cryptosporidium infection in children aged 1-6 years in XUAR, North-West China, and tracked possible sources of infection and assessed the crossspecies spread of Cryptosporidium spp. between children and animals using genotyping and subtyping tools. Such knowledge can provide insight into control and prevention strategies against infections for this pathogen.

\section{Methods}

\section{Sample Collection}

Between August 2017 to January 2019, 609 fecal samples were collected from kindergarteners aged 2-6 $y$ from 11 counties of Southern Xinjiang, China (Table 1). The parents/guardians who provided consent on behalf of their children were trained with relevant guidelines by the staff and were provided a labeled plastic fecal collector marked with the date of collection and patient identity information (age and sex). None of the kids experienced diarrhea during the sampling period. After collection, samples were stored at $4^{\circ} \mathrm{C}$. 
Table 1

Prevalence and species distribution of Cryptosporidium among children from southern Xinjiang by counties and gender

\begin{tabular}{|c|c|c|c|c|}
\hline \multirow{2}{*}{$\begin{array}{l}\text { Collection } \\
\text { site }\end{array}$} & \multicolumn{2}{|l|}{ Boy } & \multicolumn{2}{|l|}{ Girl } \\
\hline & $\begin{array}{l}\text { No. } \\
\text { Positive/No. } \\
\text { Samples } \\
\text { (\%) }\end{array}$ & Species and subtype (n) & $\begin{array}{l}\text { No. } \\
\text { Positive/No. } \\
\text { Samples } \\
\text { (\%) }\end{array}$ & $\begin{array}{l}\text { Species and } \\
\text { subtype }(n)\end{array}$ \\
\hline Baicheng & $0 / 11$ & / & $0 / 12$ & / \\
\hline Hotan & $0 / 40$ & / & $0 / 40$ & / \\
\hline Kuqa & 1/19 (5.3) & C. parvum (1), IldA15G1 (1) & $1 / 19(5.3)$ & $\begin{array}{l}\text { C. parvum (1), } \\
\text { IldA14G1 (1) }\end{array}$ \\
\hline Lop & $0 / 43$ & / & $0 / 66$ & / \\
\hline Payzawat & $1 / 14(7.1)$ & C. hominis (1), IfA12G1 (1) & $0 / 11$ & l \\
\hline Pishan & $0 / 16$ & / & $0 / 21$ & / \\
\hline Poskam & $0 / 17$ & / & $1 / 18(5.6)$ & $\begin{array}{l}\text { C. hominis (1), } \\
\text { IbA9G3 (1) }\end{array}$ \\
\hline Shufu & $0 / 26$ & / & $0 / 22$ & / \\
\hline Tumushuke & $0 / 33$ & / & $0 / 29$ & / \\
\hline Yecheng & $2 / 46(4.3)$ & C. felis (2) & $0 / 43$ & / \\
\hline Yopurga & 2/34 (5.9) & $\begin{array}{l}\text { C. hominis (1), IdA14 (1); C. } \\
\text { felis (1) }\end{array}$ & $0 / 29$ & / \\
\hline Subtotal & $6 / 299(2.0)$ & $\begin{array}{l}\text { C. felis (3); C. hominis (2), } \\
\text { IdA14 (1), IfA12G1 (1); C. } \\
\text { parvum (1), IldA15G1 (1) }\end{array}$ & $2 / 310(0.6)$ & $\begin{array}{l}\text { C. hominis (1), } \\
\text { IbA9G3 (1); C. } \\
\text { parvum (1), } \\
\text { IIdA14G1 (1) }\end{array}$ \\
\hline Total & $8 / 609(1.3)$ & $\begin{array}{l}\text { C. felis (3); C. hominis (3), lb, } \\
\text { parvum (2), IdA } 14 \mathrm{G} 1 \text { (1), lld }\end{array}$ & $\begin{array}{l}(1), \operatorname{ld} A 14(1) \\
1(1)\end{array}$ & $\mathrm{A} 12 \mathrm{G} 1(1) ; C$ \\
\hline
\end{tabular}

\section{DNA Extraction and PCR Amplification}

Approximately $200 \mathrm{mg}$ of each fecal sample was extract the genomic DNA used the E.Z.N.A® stool DNA kit, and stored the extracted DNA at $-20^{\circ} \mathrm{C}$ before PCR analysis.

We used nested PCR amplification of an approximately 830 bp partial SSU rDNA gene fragment to screen the samples for the presence of Cryptosporidium spp. We used primers described previously in a study by Xiao et al. [10]. Additionally, we used nested PCR to amplify an $850 \mathrm{bp}$ gene fragment of a $60-\mathrm{kDa}$ glycoprotein (gp60) to subtype Cryptosporidium positive isolates. This study was done using primers 
described previously in a study by Alves et al. [11]. Also, $\mathrm{dH}_{2} \mathrm{O}$ was included as a negative control and DNA from chicken-derived $C$. bailey was used as the positive control.

\section{Sequence and Phylogenetic Analyses}

We used GENEWIZ to bidirectionally sequence positive secondary PCR products. Sequencing of PCR products was performed to confirm sequence accuracy during DNA preparation. We used DNASTAR Lasergene EditSeq v7.1.0 (http://www.dnastar.com/) to edit the derived sequences and used Clustal X v2.1 (http://www.clustal.org/) to align them with reference sequences downloaded from GenBank.

A phylogenetic tree was constructed using MEGA7 using the Maximum Likelihood method based on the Tamura-Nei model to evaluate the genetic relationship between the gp60 subtypes of $C$. hominis and $C$. parvum.

\section{Results}

Prevalence of Cryptosporidium

Cryptosporidium spp. was identified in a few asymptomatic children, with only eight Cryptosporidium positive $(1.3 \%, 8 / 609)$ based on the SSU rDNA gene. The infection rate of Cryptosporidium in children in 5 of the 11 collection sites of the Xinjiang Province was: Payzawat (4.0\%; 1/25), Yopurga (3.2\%; 2/63), Yecheng (2.2\%; 2/89), Poskam (2.9\%; 1/35), and Kuqa (5.3\%; 2/38). No substantial difference in the infection rate of Cryptosporidium in children from the five sites $(P>0.05)$ (Table 1). The infection rate of Cryptosporidium was $2.0 \%$ (6/299) in boys and $0.6 \%(2 / 310)$ in girls. There was no insignificant difference in the infection rate of Cryptosporidium in boys and in girls $(P>0.05)$.

\section{Genetic characterizations at the SSU rRNA gene}

Three Cryptosporidium species were identified, including C. felis (37.5\%; 3/8), C. hominis (37.5\%; 3/8), and $C$. parvum $(25.0 \% ; 2 / 8)$. The three, three and two SSU rDNA sequences of $C$. felis, $C$. hominis and $C$. parvum were identical, respectively and showed $100 \%$ similarity with the sequences in Genbank: AF159113 for $C$. felis, MK990042 for C. hominis and MK982463 for $C$. parvum. Amongst them, $C$. hominis was identified at three collection sites (Payzawat, Poskam and Yopurga), C. felis in Yopurga and Yecheng, and $C$. parvum only in Kuqa. $C$. felis was only identified in boys, whilst $C$. hominis and $C$. parvum were both identified in both boys and girls.

Subtyping of C. hominis and C. parvum of the gp60 gene

All six $C$. hominis and $C$. parvum isolates were successfully amplified at the gp60 gene. Sequence analysis of three $C$. hominis isolates showed three subtypes, termed IbA9G3 (1), IdA14 (1) and IfA12G1 (1). The sequences of two C. parvum isolates identified in the study showed $100 \%$ similarity to those of IIdA14G1 and IIdA15G1, respectively. 


\section{Discussion}

Almost all countries in the world except Antarctica has been recorded to have a high incidence of cryptosporidiosis in humans [12]. The African nations, central and south American countries, Asian nations, European countries, and North American countries have been reported to have $2.6 \%-21.3 \%$, $3.2 \%-31.5 \%, 1.3 \%-13.1 \%, 0.1 \%-14.1 \%, 0.3 \%-4.3 \%$ cases of Cryptosporidium infection, respectively, resulting in a pooled prevalence of $7.6 \%[9,13]$. Statistics have shown that up to 2018 in China, approximately 200,054 individuals participated in Cryptosporidium related studies, of which 5,933 (3.0\%) were diagnosed with Cryptosporidium infection/cryptosporidiosis. The disease was found to be less prevalent in adults $(1.9 \%, 402 / 21316)$ than in children $<5$ years old $(2.6 \%, 269 / 10491)$ in China $(P<0.01)$ [9].

Other than age, sensitivity and specificity of the detection techniques, host health, and living criteria might also affect disease prevalence [14]. The estimated prevalence was high in those from low-income countries, individuals with gastrointestinal symptoms and residents not living in urban areas [12]. In developing countries, the prevalence of Cryptosporidium in children under five years with diarrhea was $27.4 \%$ in India (ELISA) [15], 27.8\% and 32.0\% in Ghana and Guatemala (microscopic analysis), respectively $[16,17]$, and $25.0 \%$ and $10.4 \%$ in Uganda and Tanzania (PCR), respectively $[18,19]$. In China, significantly higher prevalence of Cryptosporidium was observed in rural population (1.8\%-12.9\%) than in urban population (0-3.7\%) [9]. In the USA, cryptosporidiosis was mainly observed in children between 1-9 $y$ of age; its high incidence was associated with recreational water use and communal swimming venues, resulting in peak infection in the summer [20].

There was a limited of information on the prevalence of asymptomatic infection. Cryptosporidium was detected in 20 (7.2\%) of 276 asymptomatic aboriginal children living in villages in Malaysia [21]. In Jeddah, South Africa, almost 4.7\% Cryptosporidium-positive cases were asymptomatic compared with $32.0 \%$ cases with diarrhea from pediatric clinics [22]. This study is the first study to explore the infection of Cryptosporidium in asymptomatic children in China with low infection rates $(1.3 \% ; 8 / 609)$.

Three Cryptosporidium species were identified, including C. felis $(37.5 \% ; 3 / 8)$, C. hominis $(37.5 \% ; 3 / 8)$ and C. parvum $(25.0 \% ; 2 / 8)$. C. felis oocysts were first identified in cat feces and were therefore considered a host-adapted species [23]. Human $C$. felis infections have shown more recent prevalence in developing countries including China, where it was found to cause Cryptosporidium infection in minimum eight human cases [7]. In addition to cats and humans, $C$. felis has also been found in other animals including non-human primates, calves, horses, and foxes, suggestive of a possible risk of zoonotic transmission [24]. C. hominis is a pathogenic species commonly found in humans and natural infections have been reported in nonhuman primates, cattle, dugong, marsupials, and goats [25]. The major Cryptosporidium species in Chinese population is $C$. hominis, which has been shown to cause approximately $48.3 \%$ $(127 / 263)$ of human cases [9]. Recent studies have identified $C$. hominis as the dominant Cryptosporidium species in other species in China, such as horses and donkeys [26-28]. This supports the theory that these animals have been infected by human feces. $C$. parvum has been shown to have the 
highest zoonotic potential among the Cryptosporidium species. In China, 16.7\% (44/263) of human cases are caused by $C$. parvum [9]. In this study, we identified $C$. parvum in yaks, sheep, goats, golden takins, horses, cattle, and donkeys, indicating zoonotic potential.

Subtyping tools based on the analysis of the gp60 gene have been developed for human-pathogenic Cryptosporidium spp. to track potential sources of infection [7]. In this study, three subtype families (Ib, Id, and If) were identified for $C$. hominis, composed of IdA14, IfA12G1 and IbA9G3. Elevated frequency of all three subtype families (Ib, Id, and If) were identified in rhesus monkeys in Guizhou, where humans and animals are known to closely interact [29]. This study is the first work that is performed on humans with the discovery that those subtypes can spread between humans and monkeys.

There is a limited availability of data on the subtypes of $C$. parvum in humans in China compared to $C$. hominis. Only two subtypes (Ila and IId) have been identified and only IIdA19G1 was found [30]. In this study, subtyping successfully identified two $C$. parvum isolates of the Ild family; IldA15G1 and IIdA14G1. These were the dominant groups in animals in China indicating that these animals may represent an important source of zoonotic Cryptosporidium in China [7].

\section{Conclusions}

This study was the first to identify the presence of asymptomatic cryptosporidium infections in children in Southern Xinjiang China. Three species of Cryptospridium including C. felis, C. hominis and C. parvum were found. Meanwhile, three $C$. hominis subtypes (IdA14, IfA12G1 and IbA9G3) and two $C$. parvum subtypes (IIdA14G1 and IIdA15G1) were identified. The presence of common zoonotic $C$. parvum subtypes highlights the possible cross-species transmission of Cryptosporidium between children and animals.

\section{Abbreviations}

gp60: 60-kDa glycoprotein; SSU: small subunit

\section{Declarations}

\section{Acknowledgements}

We thank Editideas (www.editideas.cn) for its linguistic assistance during the preparation of this manuscript.

\section{Ethics approval and consent to participate}

The Research Ethics Committee and the Ethics Committee of Tarim University approved the study protocol. During the entire procedure, no children were injured.

\section{Consent for publication}


Not applicable.

\section{Availability of data and materials}

All data generated or analysed during this study are included in this published article.

\section{Competing interests}

The authors declare that they have no competing interests.

\section{Funding}

This work was supported, in part, by the National Natural Science Foundation of China (31702227), and the Program for Young and Middle-aged Leading Science, Technology, and Innovation of Xinjiang Production \& Construction Corps (2018CB034).

\section{Authors' contributions}

MQ and WZTW, ZW, YZ and QZTW and ZWTW, TW and MQ and LZ

\section{References}

1. Garcia-R JC, Hayman DT. Origin of a major infectious disease in vertebrates: The timing of Cryptosporidium evolution and its hosts. Parasitology. 2016;143:1683-1690.

2. Bouzid M, Hunter PR, Chalmers RM, Tyler KM. Cryptosporidium pathogenicity and virulence. Clin Microbiol Rev. 2013;26:115-34.

3. Platts-Mills JA, Babji S, Bodhidatta L, Gratz J, Haque R, Havt A, et al. Pathogen-specific burdens of community diarrhoea in developing countries: a multisite birth cohort study (MAL-ED). Lancet Glob Health. 2015;3:e564-75.

4. Zahedi A, Ryan U. Cryptosporidium - An update with an emphasis on foodborne and waterborne transmission. Res Vet Sci. 2020;132:500-512.

5. Ryan U, Fayer R, Xiao L. Cryptosporidium species in humans and animals: current understanding and research needs. Parasitology. 2014;141:1667-85.

6. Ježková J, Limpouchová Z, Prediger J, Holubová N, Sak B, Konečný R, et al. Cryptosporidium myocastoris sp. (Apicomplexa: Cryptosporidiidae), the Species Adapted to the Nutria (Myocastor coypus). Microorganisms. 2021;9(4):813.

7. Feng Y, Ryan UM, Xiao L. Genetic diversity and population structure of Cryptosporidium. Trends Parasitol. 2018;34:997-1011.

8. Guo Y, Cebelinski E, Matusevich C, Alderisio KA, Lebbad M, McEvoy J, et al. Subtyping novel zoonotic pathogen Cryptosporidium chipmunk genotype I. J Clin Microbiol. 2015;53:1648-54. 
9. Liu A, Gong B, Liu X, Shen Y, Wu Y, Zhang W, et al. A retrospective epidemiological analysis of human Cryptosporidium infection in China during the past three decades (1987-2018). PLoS Negl Trop Dis. 2020;14:e0008146.

10. Xiao L, Morgan UM, Limor J, Escalante A, Arrowood M, Shulaw W, et al. Genetic diversity within Cryptosporidium parvum and related Cryptosporidium Appl Environ Microbiol. 1999;65:3386-91.

11. Alves M, Xiao L, Sulaiman I, Lal AA, Matos O, Antunes F. Subgenotype analysis of Cryptosporidium isolates from humans, cattle, and zoo ruminants in Portugal. J Clin Microbiol. 2003;41:2744-7.

12. Yang X, Guo Y, Xiao L, Feng Y. Molecular epidemiology of human cryptosporidiosis in low- and middle-income countries. Clin Microbiol Rev. 2021;34:e00087-19.

13. Fayer R. Cryptosporidium: a water-borne zoonotic parasite. Vet Parasitol. 2004;126:37-56.

14. Putignani L, Menichella D. Global distribution, public health and clinical impact of the protozoan pathogen Cryptosporidium. Interdiscip Perspect Infect Dis. 2010;2010:753512.

15. Bera P, Das S, Saha R, Ramachandran VG, Shah D. Cryptosporidium in children with diarrhea: a hospital-based Study. Indian Pediatr. 2014;51:906-8.

16. Adjei AA, Armah H, Rodrigues O, Renner L, Borketey P, Ayeh-Kumi P, et al. Cryptosporidium, a frequent cause of diarrhea among children at the Korle-Bu Teaching Hospital, Accra, Ghana. Jpn J Infect Dis. 2004;57:216-9.

17. Laubach HE, Bentley CZ, Ginter EL, Spalter JS, Jensen LA. A study of risk factors associated with the prevalence of Cryptosporidium in villages around Lake Atitlan, Guatemala. Braz J Infect Dis. 2004;8:319-23.

18. Tumwine JK, Kekitiinwa A, Nabukeera N, Akiyoshi DE, Rich SM, Widmer G, et al. Cryptosporidium parvum in children with diarrhea in Mulago Hospital, Kampala, Uganda. Am J Trop Med Hyg. 2003;68:710-5.

19. Tellevik MG, Moyo SJ, Blomberg B, Hjøllo T, Maselle SY, Langeland N, et al. Prevalence of Cryptosporidium parvum/hominis, Entamoeba histolytica and Giardia lamblia among young children with and without diarrhea in Dar es Salaam, Tanzania. PLoS Negl Trop Dis. 2015;9:e0004125.

20. Barry MA, Weatherhead JE, Hotez PJ, Woc-Colburn L. Childhood parasitic infections endemic to the United States. Pediatr Clin North Am. 2013;60:471-85.

21. Al-Mekhlafi HM, Mahdy MA, 'Azlin MY, Fatmah MS, Norhayati M. Childhood Cryptosporidium infection among aboriginal communities in Peninsular Malaysia. Ann Trop Med Parasitol. 2011;105:135-43.

22. Al-Braiken FA, Amin A, Beeching NJ, Hommel M, Hart CA. Detection of Cryptosporidium amongst diarrhoeic and asymptomatic children in Jeddah, Saudi Arabia. Ann Trop Med Parasitol. 2003;97:505-10.

23. Iseki M. Cryptosporidium felis n. (Protozoa Eimeriorina) from the domestic cat. Jap J Parasitol. 1979;28:285-307. 
24. Jiang W, Roellig DM, Lebbad M, Beser J, Troell K, Guo Y, et al. Subtype distribution of zoonotic pathogen Cryptosporidium felis in humans and animals in several countries. Emerg Microbes Infect. 2020;9:2446-2454.

25. Zhao W, Zhou H, Jin H, Liu M, Qiu M, Li L, et al. Molecular prevalence and subtyping of Cryptosporidium hominis among captive long-tailed macaques (Macaca fascicularis) and rhesus macaques (Macaca mulatta) from Hainan Island, southern China. Parasit Vectors. 2019;12:192.

26. Jian F, Liu A, Wang R, Zhang S, Qi M, Zhao W, et al. Common occurrence of Cryptosporidium hominis in horses and donkeys. Infect Genet Evol. 2016;43:261-6.

27. Wang W, Zhang Z, Zhang Y, Zhao A, Jing B, Zhang L, et al. Prevalence and genotypic identification of Cryptosporidium in free-ranging and farm-raised donkeys (Equus asinus asinus) in Xinjiang, China. Parasite. 2020;27:45.

28. Li F, Su J, Chahan B, Guo Q, Wang T, Yu Z, et al. Different distribution of Cryptosporidium species between horses and donkeys. Infect Genet Evol. 2019;75:103954.

29. Ye J, Xiao L, Ma J, Guo M, Liu L, Feng Y. Anthroponotic enteric parasites in monkeys in public park, China. Emerg Infect Dis. 2012;18:1640-3.

30. Yu F, Li D, Chang Y, Wu Y, Guo Z, Jia L, et al. Molecular characterization of three intestinal protozoans in hospitalized children with different disease backgrounds in Zhengzhou, central China. Parasit Vectors. 2019;12:543.

\section{Figures}




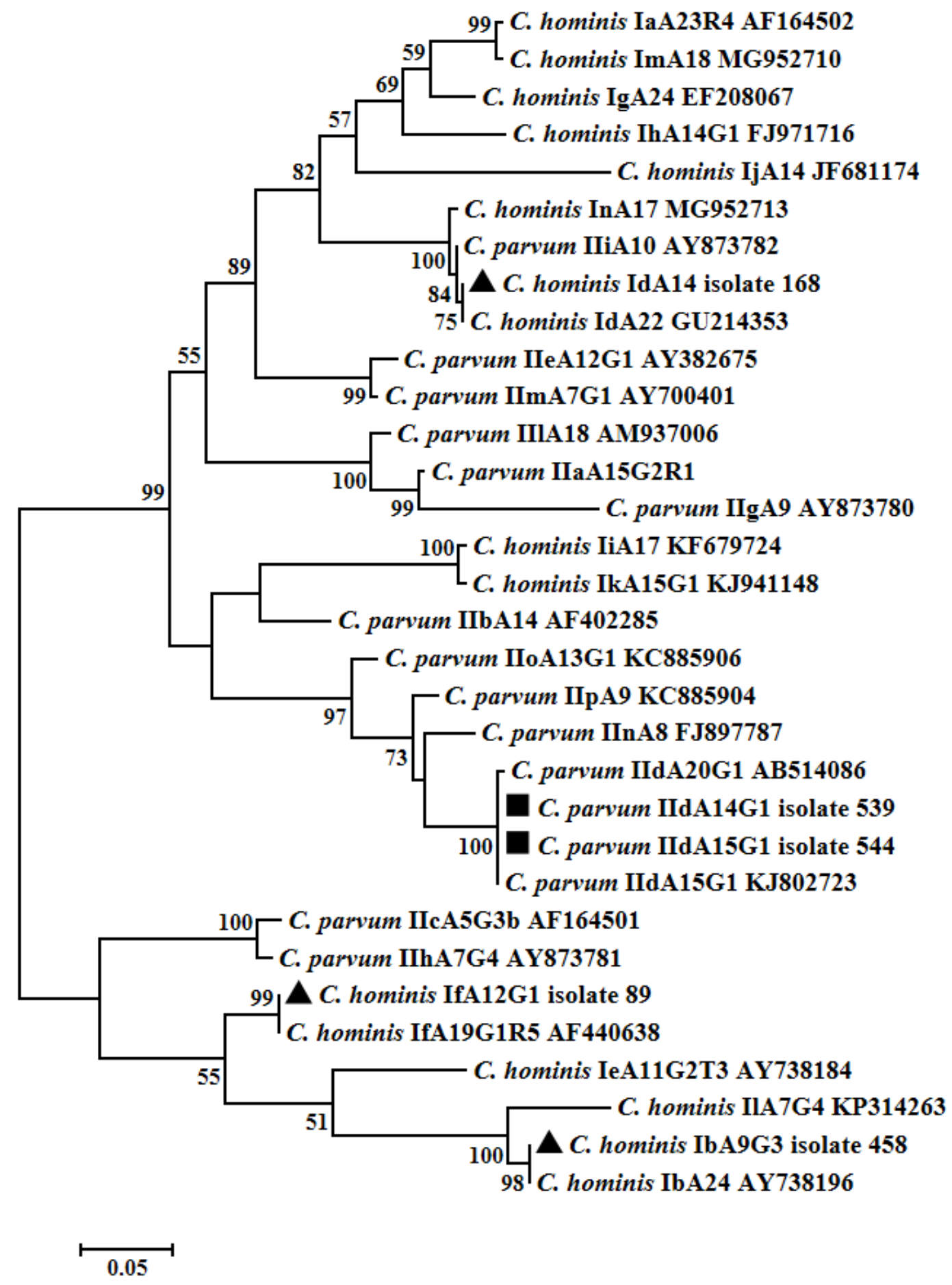

Figure 1

Molecular Phylogenetic analysis. The Tamura-Nei model-based Maximum Likelihood method was used to determine the evolutionary history. Here is the tree with the highest log likelihood with branches showing the \% of trees with clusters of relevant taxa. The NJ and BioNJ algorithms were used to obtain the initial tree(s) for heuristic searches using the MCL method. We selected the topology with a superior log likelihood value. The tree has been drawn to scale, and the number of substitutions per-site indicate 
branch lengths. There were 32 nucleotide sequences used for analysis. Codon positions included 1 st + 2nd $+3 r d+$ non-coding. All positions with missing data and gaps were excluded, resulting in 292 positions. MEGA7 was used to perform the evolutionary analyses.

\section{Supplementary Files}

This is a list of supplementary files associated with this preprint. Click to download.

- GraphicalAbstract.tif 